\title{
Tratamiento de un priapismo de alto flujo postraumático
}

\author{
Treating a traumatic high-flow priapism
}

Juan M. Pereira' ${ }^{1}$ Alejandro M. Farah'1, Juan J. Pissani²

\section{RESUMEN}

La incidencia del priapismo de etiología traumática ha aumentado. El priapismo de alto flujo (PAF), hecho el diagnóstico, es una urgencia diferida pasible de resolución vía hemodinamia con embolización selectiva de la fístula si fracasaron los métodos clásicos de resolución. El uso de spongostan como vehículo embolizante es factible seguro y económico. El objetivo de este artículo es analizar el caso de un paciente que consulta por priapismo, el algoritmo seguido y la resolución final del caso.

Palabras claves: priapismo de alto flujo, fistula arteriocavernosa, embolización selectiva.

\begin{abstract}
The incidence of traumatic etiology priapism has increased. The high-flow priapism (PAF), afther the diagnosis, is a medical emergency delayed resolution via hemodynamics with selective embolization of the fistula may, if they failed the classical methods of resolution. The use of spongostan as a vehicle embolization is feasible, safe and economic. The aim of this paper is to analyze the case of a patient who consulted by the priapism, followed algorithm and the final resolution of the case.
\end{abstract}

Revista Argentina de Cardioangiología Intervencionista 2014;5(2):147-149

\section{INTRODUCCIÓN}

El priapismo se define como una erección persistente patológica del pene, no asociada a la estimulación sexual, consecuencia de un desequilibrio entre los flujos arterial de entrada y venoso. ${ }^{1-4}$

El estado de erección se limita a los cuerpos cavernosos, sin afectar al cuerpo esponjoso o el glande como ocurre en la erección fisiológica. ${ }^{1} \mathrm{La}$ incidencia del priapismo, si bien es baja, ha aumentado. Su etiología traumática desde la introducción de las inyecciones intracavernosas de fármacos vasoactivos pasó a ser una entidad de aparición relativamente frecuente. El tratamiento por catéter es siempre de "urgencia diferida, por el peligro de fibrosis de los cuerpos cavernosos y la subsiguiente impotencia”., ${ }^{1,8}$

\section{CASO CLÍNICO}

Paciente de 31 años, que ingresa por guardia del Hospital San Bernardo, con priapismo no doloroso, de 8

\footnotetext{
1. Servicio de Hemodinamia.

2. Servicio de Urología.

Hospital San Bernardo, Salta Capital, Argentina

$\bowtie$ Correspondencia: Juan Manuel Pereira. Ameghino 243, Salta Capital, Salta, Argentina|juanm_tucu@yahoo.com.es
}

Conflictos de intereses: no existen. horas de evolución. Refiere un traumatismo pélvico en bicicleta, tres días antes. Se realiza, en Guardia, punción en la base de ambos cuerpos cavernosos, con trocar abbocath $\mathrm{N}^{\circ} 14$ y a continuación, inyección intracavernosa con etilefrina, sin resolución persistente de la patología. Posteriormente, en quirófano, se practica un shunt cavernoso esponjoso que mejora el cuadro, pero 4 horas más tarde reaparecen los signos del priapismo. Se sospecha priapismo de alto flujo, sin isquemia, por fistula arterio venosa. Se decide intentar su embolización selectiva. Por punción femoral derecha, por introductor $6 \mathrm{Fr}$, se avanzó catéter diagnóstico de 4 F. Se realizó arteriografía selectiva con contraste no iónico, de arteria ilíaca interna izquierda. Se localiza arteria pudenda interna derecha y se cateteriza en forma selectiva y distal de la misma. Se constata fistula arteriocavernosa de alto flujo (Figura 1) y se emboliza con spongostan (Figura 2). Posteriormente, el paciente refiere mejoría paulatina. A las 16 horas del procedimiento se constata pene sin erección. En el seguimiento, a la semana, el paciente refiere dos erecciones normales.

\section{DISCUSIÓN}

En la clasificación del priapismo encontramos 2 grupos, alto y bajo flujo. ${ }^{2-4}$

El priapismo de bajo flujo ( $\mathrm{PBF})$, de estasis o isquémico, es más frecuente y entraña mayor potencial de causar disfunción eréctil. Hay una obstrucción, parcial o 


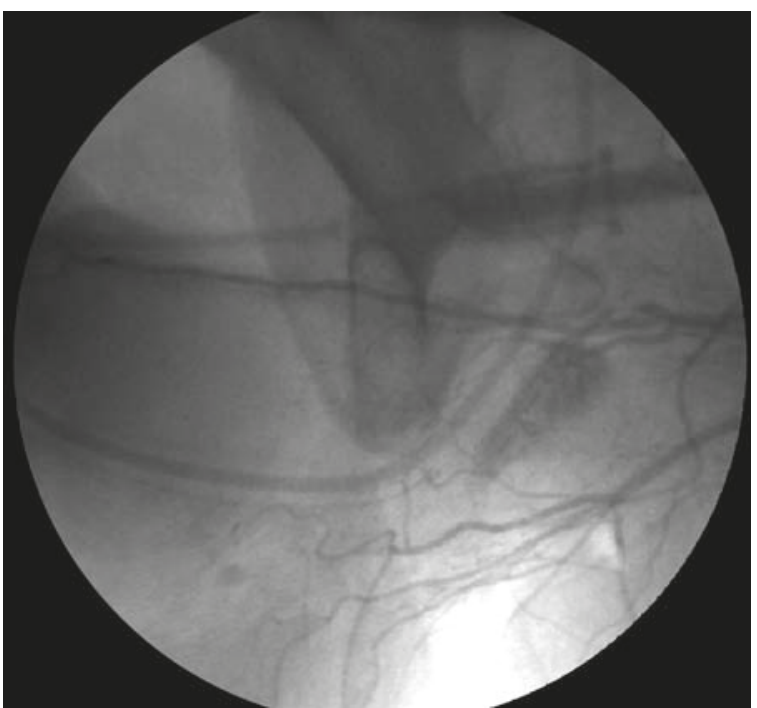

Figura 1.

completa, del drenaje de los cuerpos cavernosos. Instaurada la isquemia, la $\mathrm{pO}_{2}$ y el $\mathrm{pH}$ de la sangre atrapada en los cuerpos cavernosos disminuyen, a niveles de hipoxia y acidosis severa en 3-4 horas. El estado isquémico prolongado lleva a la muerte celular y fibrosis. El paciente acude con una erección completa, dolorosa, de varias horas de duración.

El priapismo de alto flujo (PAF) se define por un aumento del flujo sanguíneo arterial en los cuerpos cavernosos, con una erección mantenida con buena oxigenación tisular. Presenta drenaje venoso normal, pero insuficiente para provocar la detumescencia. Suele ser provocado por una fístula arteriocavernosa adquirida -yatrogénica o accidental- asociados a un traumatismo arterial o peneano con laceración de la arteria cavernosa o una de sus ramas. Precisan más tiempo para instaurarse, con una evolución más favorable para el mantenimiento posterior de la función sexual. Refieren un trauma, desde horas hasta días antes. Cuando es por trauma penetrante, en general por aguja, se instaura de inmediato. Suele ser indolora o con algunas molestias. El pene presenta una erección incompleta, no doloroso al tacto, y de consistencia elástica. ${ }^{1}$

En la evaluación, la gasometría de la punción-aspiración de sangre de los cuerpos cavernosos revela, en el PAF, características normales del flujo arterial. En el PBF hay hipoxia y acumulación de metabólicos ácidos. Por Doppler se comprueba un incremento del flujo arterial en el PAF, mientras que en el PBF no existe dicho incremento. Permite la visualización y localización de la fístula arteriolacunar. ${ }^{5}$

La cavernosografía demuestra la aparición de un flujo venoso normal tras la inyección de contraste en los cuerpos cavernosos en el PAF. En el PBF el flujo de salida del contraste es lento y está disminuido. ${ }^{1}$

La gammagrafía demuestra, en el PAF, cómo el 99 Tc intravenoso o intracavernoso se distribuye regularmente por ambos cuerpos cavernosos. En la PBF, la di-

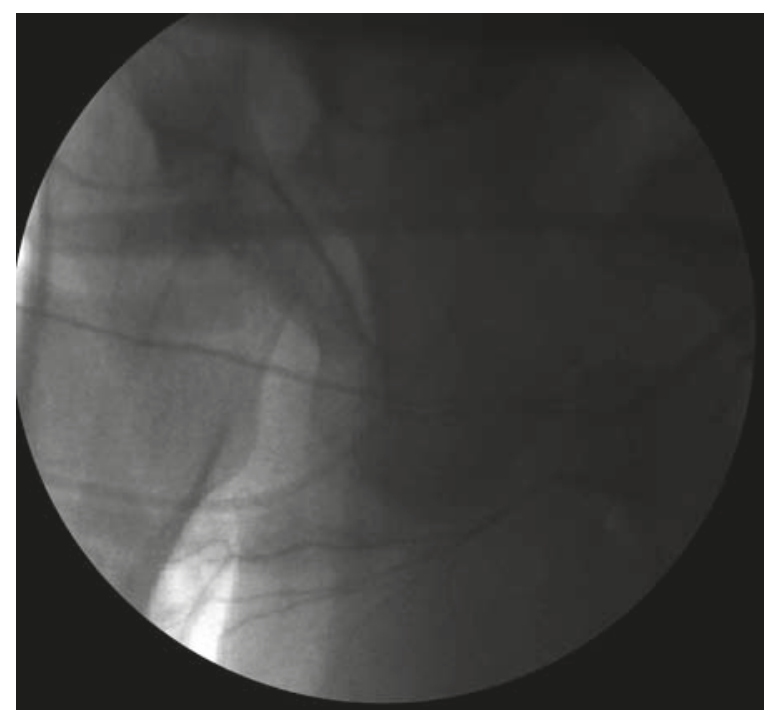

Figura 2.

fusión del isótopo está disminuida. El uso de resonancia magnética nuclear puede evidenciar la o las fístulas preembolización. ${ }^{6}$

El tratamiento del priapismo debe ser considerado como una urgencia, diferida en el PAF e inmediata en el PBF. ${ }^{1,4}$

La interrupción de los fármacos asociados con el priapismo (antihipertensivos, antipsicóticos, nutrición parenteral), la sedación, la analgesia y la hidratación, deben ser la norma.

Si se debe a inyección intracavernosa de fármacos vasoactivos, se recomienda la realización de ejercicio físico durante 30 minutos. De persistir, proceder a la punción-aspiración en la cara lateral de los cuerpos cavernosos, la inyección intracavernosa de fármacos agonistas $\alpha 1$-adrenérgicos y la punción-aspiración bilateral. ${ }^{7.8}$ La arteriografía no se debe utilizar con fines diagnósticos, sino terapéuticos y solo para el priapismo de alto flujo. ${ }^{1,3} \mathrm{El}$ acceso por arteria femoral, con técnica de crossover, se llega hasta la arteria ilíaca interna contralateral. Se intenta identificar la arteria pudenda interna, que nace a cada lado de la división anterior de la arteria ilíaca interna y porque atraviesa el agujero obturador. En el caso del PAF, con la arteriografía selectiva se aprecia la opacificación de los cuerpos cavernosos por el contraste proveniente del sistema arterial. En el PBF no se visualizan las arterias cavernosas, sólo las dorsales y bulbouretrales.

La fístula normalmente es unilateral, a la vez que la embolización unilateral se realiza para reducir el riesgo de gangrena cavernosa y disfunción eréctil. Se debe evitar el vasoespasmo, pudiéndose usar vasodilatadores como la nitroglicerina $(10 \mathrm{~mm} / \mathrm{ml})$, que de paso ayudará a tener una mejor angiografía. El enrojecimiento normal del cuerpo cavernoso puede aparecer localizado e indicar el punto de la hemorragia, aunque suele presentar hematomas y equimosis por las intervenciones previas. Localizada la arteria pudenda, se eligen 
proyecciones oblicuas, para obtener proyecciones ortogonales del pene. La embolización se debería realizar en una posición altamente selectiva y lo más cerca del punto de hemorragia, lo que evitaría las complicaciones isquémicas, conservaría el aporte sanguíneo de los cuerpos cavernosos y la función eréctil.

Los materiales utilizados para la embolización los podríamos dividir entre reabsorbibles e irreabsorbibles. Dentro de los primeros se ha utilizado el coágulo autólogo y la esponja de gelatina. La embolización con $3 \mathrm{ml}$ de coágulo autólogo es efectiva. ${ }^{4}$ Estos son reabsorbidos con el tiempo, con recanalización de la arteria embolizada y recuperación completa de la capacidad eréctil. Se puede seguir mediante sonografía con Doppler color. En las fístulas grandes, se puede emplear esponja de gelatina y los microespirales. Entre los materiales no-reabsorbibles se ha utilizado el bucrilato y los espirales metálicos (coils de platino), que dan una embolización definitiva y parecieran tener una recuperación de la capacidad eréctil igualmente buena. ${ }^{1,3}$

Después de la embolización, se debe visualizar el lado contralateral. Si presenta fistula, se puede tomar conducta expectante, y si resulta sintomática, embolizarla posteriormente. En los casos de fístula bilateral, ini-

\section{BIBLIOGRAFÍA}

1. Rodríguez Tolrá J, Cuadrado Campaña JM, Ropero Valverde J, Ruiz Salas $V$, Franco Miranda E. Nuestra experiencia en el priapismo de alto flujo por fístula arterio-lacunar uni-y bilateral. Actas Urol Esp 2007;31(2):113-119.

2. Ibáñez Suárez R, Barranco Medina J, Flores Ortiz J, Arroyo Maestre JM, Querol Canteras A. Emergencias urológicas 2: Síndrome escrotal agudo. Priapismo. Capítulo 7.5. En: Principios de urgencias, emergencias y cuidados críticos.

3. Burke CT, Dixon RG, Mauro MA, et al (eds). Radiología de alto rendimiento. Tratamiento del priapismo de alto flujo. Editorial Elsevier 2011;338-340.

4. Sancak T, Conkbayir I. Post-traumatic high-flow priapism: management by superselectivetranscatheter autologous clot embolization and duplex sonography-guided compression. J Clin Ultrasound 2001;29(6):349-353.

5. Bertolotto M, Quaia E, Mucelli FP, Ciampalini S, Forgács B, Gattuccio I. Color Doppler imaging of posttraumatic priapism before and after selective embolization. Radiographics 2003;23(2):495-503 cialmente se abogaba para que se realice en dos tiempos, con material reabsorbible y con una diferencia de varias semanas, esperando a que la primera arteria embolizada se recanalizase. Algunos toman posturas conservadoras con la segunda fístula, convirtiéndose, si se resuelve, en un proceso de embolización unilateral. Algunas experiencias hablan de la factibilidad y seguridad de la embolización en un solo tiempo de ambas.

La embolización es efectiva en el 79\% de los casos y la repermeabilización aparece en el 10 a 30\% de ellos, que se trata repitiendo la embolización. La función eréctil se conserva en el 80 a $90 \%$ de los pacientes, tras la embolización, y puede tardar entre 2 a 3 meses. ${ }^{1,3}$

Existen técnicas de tratamiento quirúrgicas de derivación, en la fase de isquemia avanzada, cuando los métodos terapéuticos han fracasado, creando cortocircuitos.

\section{CONCLUSIONES}

El PAF es una urgencia poco frecuente, pero en aumento. Su tratamiento, de urgente pero diferido, es pasible de resolución vía hemodinamia con embolización selectiva de la fístula, factible y seguro.

6. White C, Gulati M, Gomes A, Rajfer J, Raman S. Pre-embolization evaluation of high-flow priapism: magnetic resonance angiography of the penis. Abdom Imaging. 2013 Jun;38(3):588-97. doi: 10.1007/s00261-012-9936-9.

7. Burnett AL, Sharlip ID. Standard operating procedures for priapism. J SexMed.2013 Jan;10(1):180-94. doi: 10.1111/j.1743-6109.2012.02707.x. Epub 2012 Mar 29.

8. Mizutani M, Nakano H, Sagami K, Nihira H. Treatment of post-traumatic priapism by intracavernosus inyection of alpha-stimulant. Urol Int 1986:41(4):312-314

9. Pagà Carbonell J, Peri i Cusi L. Priapismo de alto flujo y larga evolución Presentación de un caso y propuesta de algoritmo diagnóstico y tratamiento. Actas Urol Esp 2005;29(7):708-710.

10. Görich J, Ermis C, Krämer SC, Fleiter T, Wisianowsky C, Basche S, Gottfried $H W$, Volkmer BG. Interventional treatment of traumatic priapism. J Endovasc Ther 2002;9(5):614-617 